\title{
Levodopa withdrawal syndrome identical to neuroleptic malignant syndrome
}

\author{
W.R.G. Gibb and D.N.W. Griffith \\ University College Hospital, London WC1E6AU, UK.
}

\begin{abstract}
Summary: A 60 year old woman with idiopathic Parkinson's disease had been prescribed thioridazine for schizophrenia. Five months after this was stopped, Sinemet also considered of dubious therapeutic value, was withdrawn. One week later she developed features of the neuroleptic malignant syndrome (NMS) accompanied by myoglobinuric renal failure. Post-mortem examination confirmed Lewy body degeneration in the substantia nigra. It is proposed that NMS may be caused by levodopa withdrawal in Parkinson's disease, and that it is withdrawal of dopaminergic drive that causes the syndrome.
\end{abstract}

\section{Introduction}

The neuroleptic malignant syndrome (NMS) is an uncommon complication of treatment with neuroleptic drugs first described in the late 1950s. Characteristically it begins within days of starting or altering medication, with fever, rigidity, altered consciousness, autonomic signs and elevated creatine kinase levels. Coma may precede death from respiratory failure or cardiovascular collapse. The syndrome has not been widely recognized as a complication of alternative causes of impaired dopaminergic transmission. We describe a patient with idiopathic Parkinson's disease in whom the features of a levodopa withdrawal syndrome were identical to NMS.

\section{Case report}

A woman of 44 years developed idiopathic Parkinson's disease and the following year schizophrenia with depressive features. At the age of 51 she was given Sinemet 275 (levodopa $250 \mathrm{mg}$, carbidopa $25 \mathrm{mg}$ ) four daily, benzhexol $(6 \mathrm{mg})$, thioridazine $(50 \mathrm{mg})$ and amitriptyline $(75 \mathrm{mg})$. At the age of 60 she became depressed, failed to eat, and was admitted to hospital. She exhibited mild dementia, facial hypomimia, orofacial dyskinesias and marked axial and limb rigidity. Thioridazine and amitriptyline were stopped and she was treated with Sinemet 275 six daily and a course of ten electroconvulsive therapy sessions over one month. There was little benefit and after 4 months

Correspondence: W.R.G. Gibb, M.B., M.R.C.P., National Hospitals for Nervous Diseases, Maida Vale Hospital, London W9 1TL, UK.

Accepted: 17 July 1985 clomipramine (75 mg) and tryptophan (Pacitron $1 \mathrm{~g}$ ) were started, and the Sinemet reduced to three tablets daily. One month later it was reduced to two daily, and a week later it was stopped and orphenadrine $(150 \mathrm{mg})$ commenced.

Six days later she became drowsy, with an oral temperature of $39.5^{\circ} \mathrm{C}$, sweating, tachypnoea, pulse $160 / \mathrm{min}$, blood pressure $90 / 60 \mathrm{mmHg}$ and severe generalized rigidity. Following transfer to this hospital the axillary temperature was $40^{\circ} \mathrm{C}$, there was trismus, abdominal rigidity, and no movements either spontaneously or in response to pain. Focal neurological signs were absent and oliguria was noted. Initial investigations showed a blood urea of $25 \mathrm{mmol} / \mathrm{l}$, sodium $170 \mathrm{mmol} / \mathrm{l}$ and creatinine of $337 \mu \mathrm{mol} / \mathrm{l}$. Fluid infusions were given but oliguria persisted even after intravenous frusemide. An initial diagnosis of septicaemia with acute renal failure was considered but urine and blood cultures remained negative. On the second day four Sinemet 275 tablets were given by nasogastric tube and on the third day $6 \mathrm{mg}$ benzhexol. The creatine kinase was 7671 IU/1 (normal 24-195), alanine transaminase $900 \mathrm{IU} / 1$ (normal 7-45), aspartate transaminase $3030 \mathrm{IU} / 1$ (normal 9-41), hydroxybutyrate dehydrogenase $1072 \mathrm{IU} / 1$ (normal 55-140), $\gamma$-glutamyl transferase $20 \mathrm{IU} / 1$ (normal <65), and alkaline phosphatase $84 \mathrm{IU} / \mathrm{l}$ (normal 35-105). Fever remained above $38^{\circ} \mathrm{C}$ for 4 days and resolved 7 days later, but coma persisted. In view of her previous medical history and severe disabilities, dialysis for the acute renal failures was not started. It was following death 10 days later that the clinical similarities with NMS were first considered. At post-mortem no site of infection was found. Sections of rectus abdominis showed marked rhabdomyolysis, with similar changes 
in vastus lateralis. Kidney sections showed uniform necrosis of proximal renal tubules, but cortical tissue showed good preservation. The brain findings which confirmed idiopathic Parkinson's disease showed neuronal loss with numerous Lewy bodies in the substantia nigra, other brain stem nuclei and the basal nucleus of Meynert. Lewy bodies, widely considered to be a sign of neuronal loss, were also present in the hypothalamus.

\section{Discussion}

This patient had advanced idiopathic Parkinson's disease with severe rigidity and dementia. When Sinemet was withdrawn she developed pyrexia, increased rigidity and biochemical evidence of muscle necrosis, the cardinal triad of NMS. However, due to her recent incapacity there was severe water depletion with hypernatraemia, which on tenuous evidence is postulated as a trigger for NMS, but which could contribute to myoglobinuria. Theoretically, the tryptophan may have lead to an increase in rigidity and orphenadrine may have impaired normal heat loss mechanisms.

In 1981 features of NMS were reported in a 59 year old man, with idiopathic Parkinson's disease and a psychiatric disorder treated with haloperidol and lithium, in whom levodopa was discontinued (Henderson \& Wooten, 1981). Similar features also developed in a patient with Huntington disease treated with dopamine depleting drugs - methyltyorsine and tetrabenazine (Burke et al., 1981), and in two patients with idiopathic Parkinson's disease in whom levodopa was withdrawn (Toru et al., 1981; Sechi et al., 1984).

The specific pathological features of idiopathic Parkinson's disease, as seen in this case, include neuronal loss in the substantia nigra and hypothalamus (Langston \& Forno, 1978). The effect of with-

\section{References}

BURKE, R.E., FAHN, S., MAYEUX, R., WEINBERG, H., LOUIS, K. \& WILLNER, J.H. (1981). Neuroleptic malignant syndrome caused by dopamine-depleting drugs in a patient with Huntington disease. Neurology (NY), 31, 1022.

HENDERSON, V.W. \& WOOTEN, G.F. (1981). Neuroleptic malignant syndrome: a pathogenetic role for dopamine receptor blockade? Neurology (NY), 31, 132.

LANGSTON, J.W. \& FORNO, L.S. (1978). The hypothalamus in Parkinson's disease. Annals of Neurology, 3, 129.

DE ROHAN CHABOT, P., ELKHARRAT, D., CONSO, F., BISMUTH, Сн., \& GOULON, M. (1982). Syndrome malin des drawal of levodopa in Parkinson's disease would leŝ ve these areas substantially depleted or devoid of dopaminergic drive. It has been suggested that NMS is gue to the consequence of neuroleptic-induced dopamine receptor blockade in the basal ganglia leading to increased rigidity and heat production, and in the hypothalamus producing failure of dopamine-indueed heat loss mechanisms (Henderson \& Wooten, 19 $\overline{\mathcal{Y}}$ ). However dopamine depleting drugs and levodgpa withdrawal can cause NMS so it seems likely it is oue to a sudden reduction in dopaminergic drive at these sites.

Levodopa drug holidays have lost favour in the standard treatment of idiopathic Parkinson's diseasse because of the temporary benefit of such manipulation and occasional severe akinetic crises that risk cormplications such as inhalation and pneumonia. Akinetic crises may be encountered in other situations suclifas when levodopa is withdrawn during changes in therapy or in the management of confusional statesuor psychoses. NMS should be recognized as a potenisal consequence of a severe levodopa withdrawal regction. It may be more likely to occur in advanced disease or if it is abruptly withdrawn. Wider recognition of this disorder is required. In moderate or severe cases intensive therapy should be swiftly started using evaporative techniques for body cooling, intravenous rehydration and, when severe, with dantrolege copin combination with levodopa or dopamine agowists. Anticholinergic drugs have been recommende they are usually ineffective for the rigidity of NMS and they do not beneficially affect the hyperthermia fde Rohan Chabot et al., 1982).

\section{Acknowledgements}

We thank Dr D.P. Brenton for permission to report this 夀e and Dr A.J. Lees for helpful criticism.

neuroleptiques. Action benefique du dantrolene §ur l'hyperthermie et la rigidite musculaire. La Nouvelle PrQgse Medicale, 11, 1067.

SECHI, G.P., TANDA, F. \& MUTANI, R. (1984). Fuglal hyperpyrexia after withdrawal of levodopa. Neurology (NY), 34, 249.

TORU, M., MATSUDA, O., MAKIGUCHI, K. \& SUGANO,JK. (1981). Neuroleptic malignant syndrome-like state follow ing a withdrawal of antiparkinsonian drugs. Journadof Nervous and Mental Disease, 169, 324. 